\title{
Search for doubly charged Higgs bosons with the ATLAS detector
}

\author{
Giulia Ucchielli* on behalf of the ATLAS Collaboration \\ Technische Universität Dortmund \\ E-mail: giulia.ucchielli@cern.ch
}

Doubly charged Higgs bosons $\left(H^{ \pm \pm}\right)$appear in several beyond the Standard Model extensions, aimed to explain the mechanism for neutrino mass generation. The $H^{ \pm \pm}$either decays into pairs of leptons or $W$ bosons being, in either case, of same sign. Two complementary searches are performed using the ATLAS detector, exploring the two stated $H^{ \pm \pm}$decay patterns. This contribution discusses the methodologies used in the two searches and, eventually, the results obtained from data collected by the ATLAS detector at a center-of-mass energy of $\sqrt{s}=13 \mathrm{TeV}$.

Prospects for Charged Higgs Discovery at Colliders - CHARGED2018

25-28 September 2018

Uppsala, Sweden

${ }^{*}$ Speaker. 


\section{Doubly charged Higgs bosons}

Doubly charged Higgs bosons $\left(H^{ \pm \pm}\right)$appear in many extensions of the Standard Model, such as left-right symmetric models (LRSM) [1], Higgs triplets (HTM) [2, 3], Zee-Babu [4, 5] or GeorgiMachacek [6] models. Here we concentrate on the phenomenology of LRSM and HTM, which both introduce a Higgs boson triplet containing $H^{ \pm \pm}$and which were investigated by the ATLAS experiment [7]. Neutrino mass generation happens through the See-Saw mechanism. In LRSM right-handed counterparts for fermions, as well as for $Z$ and $W$ bosons, appear and restore the symmetry in weak interactions at energy scales of $\sim \mathrm{TeV}$.

\subsection{Production and decay}

The dominant production mode for $H^{ \pm \pm}$at the LHC is Drell-Yan pair production, as shown in Figure 1. The cross-section for this process depends on the model and on the mass of the $H^{ \pm \pm}$. It varies between $\sim 10^{-3}-10 \mathrm{fb}$, decreasing rapidly with $m_{H^{ \pm \pm}}$. Single $H^{ \pm \pm}$production via vector boson fusion or $H^{ \pm \pm}$associated production with $H^{ \pm}$have a lower cross-section compared to pair production, and are here neglected [8].

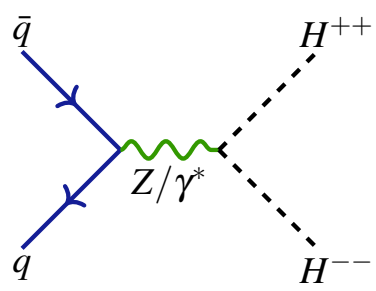

Figure 1: Doubly charged higgs boson pair production via Drell-Yan.

The branching ratio for $H^{ \pm \pm}$depends on the vacuum expectation value (VEV) of the Higgs triplet ${ }^{1}$ containing $H^{ \pm \pm}$. For very small values of the VEV $H^{ \pm \pm}$decay into pairs of leptons, while for larger values of the VEV the main decay mode is $H^{ \pm \pm} \rightarrow W^{ \pm} W^{ \pm}$. In the following, only decays into light leptons $(e, \mu)$ are considered, either originating from $H^{ \pm \pm}$or $W^{ \pm}$.

\section{Search for $\boldsymbol{H}^{ \pm \pm} \rightarrow \boldsymbol{\ell}^{ \pm} \boldsymbol{\ell}^{ \pm}$}

The analysis presents a clean signature composed by two-to-four leptons [9]. The $4 \ell$ final state has an efficiency $\times$ acceptance $(A \times \varepsilon)$ of $57.2 \%$, followed by $33.7 \%$ in the $3 \ell$ final state and $9.1 \%$ in the $2 \ell$ channel. Table 1 shows the definition of the analysis regions. Each signal region is defined by the presence of at least one same-sign lepton pair; the kinematics of the pair is exploited to set a lower cut on the pair $p_{\mathrm{T}}>100 \mathrm{GeV}$ and $\Delta R<3.5$. When two same-sign pairs are present in the event, an optimised mass-equality requirement is used to select signal events. The major background for this topology arises from misreconstructed objects: electron charge misidentification and nonprompt (also referred to as fake) leptons. The latter component is identified with leptons which originate from secondary decays of particles inside jets, wrongly reconstructed as coming from the

\footnotetext{
${ }^{1}$ The VEV is bounded by $\rho=M_{W_{L}}^{2} / \cos ^{2} \theta_{W} M_{Z}^{2}$ precision measurements to be $<1 \mathrm{GeV}$.
} 
Table 1: Summary of all analysis regions [9].

\begin{tabular}{|c|c|c|c|c|c|c|c|c|c|}
\hline \multirow{2}{*}{ Channel $\quad$ Region } & \multicolumn{3}{|c|}{ Control Regions } & \multicolumn{3}{|c|}{ Validation Regions } & \multicolumn{3}{|c|}{ Signal Regions } \\
\hline & OCCR & DBCR & $4 \mathrm{LCR}$ & SCVR & $3 \mathrm{LVR}$ & $4 \mathrm{LVR}$ & $1 \mathrm{P} 2 \mathrm{~L}$ & 1P3L & $2 \mathrm{P} 4 \mathrm{~L}$ \\
\hline Electron channel & $e^{ \pm} e^{\mp}$ & $e^{ \pm} e^{ \pm} e^{\mp}$ & & $e^{ \pm} e^{ \pm}$ & $e^{ \pm} e^{ \pm} e^{\mp}$ & & $e^{ \pm} e^{ \pm}$ & $e^{ \pm} e^{ \pm} e^{\mp}$ & \\
\hline Mixed channel & - & $e^{ \pm} \mu^{ \pm} \ell^{\mp}$ & $\begin{array}{l}\ell^{ \pm} \ell^{ \pm} \\
\ell^{\mp} \ell^{\mp}\end{array}$ & $e^{ \pm} \mu^{ \pm}$ & $\begin{array}{l}e^{ \pm} \mu^{ \pm} \ell^{\mp} \\
\ell^{ \pm} \ell^{ \pm} \ell^{\prime \mp}\end{array}$ & $\begin{array}{l}\ell^{ \pm} \ell^{ \pm} \\
\ell^{\mp} \ell^{\mp}\end{array}$ & $e^{ \pm} \mu^{ \pm}$ & $\begin{array}{l}e^{ \pm} \mu^{ \pm} \ell^{\mp} \\
\ell^{ \pm} \ell^{ \pm} \ell^{\prime \mp}\end{array}$ & $\begin{array}{l}\ell^{ \pm} \ell^{ \pm} \\
\ell^{\mp} \ell^{\mp}\end{array}$ \\
\hline Muon channel & - & $\mu^{ \pm} \mu^{ \pm} \mu^{\mp}$ & & $\mu^{ \pm} \mu^{ \pm}$ & $\mu^{ \pm} \mu^{ \pm} \mu^{\mp}$ & & $\mu^{ \pm} \mu^{ \pm}$ & $\mu^{ \pm} \mu^{ \pm} \mu^{\mp}$ & \\
\hline$m\left(e^{ \pm} e^{ \pm}\right)[\mathrm{GeV}]$ & {$[130,2000]$} & {$[90,200)$} & & {$[130,200)$} & {$[90,200)$} & & {$[200, \infty)$} & {$[200, \infty)$} & \\
\hline$m\left(\ell^{ \pm} \ell^{ \pm}\right)[\mathrm{GeV}]$ & - & {$[90,200)$} & {$[60,150)$} & {$[130,200)$} & {$[90,200)$} & {$[150,200)$} & {$[200, \infty)$} & {$[200, \infty)$} & {$[200, \infty)$} \\
\hline$m\left(\mu^{ \pm} \mu^{ \pm}\right)[\mathrm{GeV}]$ & - & {$[60,200)$} & & {$[60,200)$} & {$[60,200)$} & & {$[200, \infty)$} & {$[200, \infty)$} & \\
\hline$b$-jet veto & $\checkmark$ & $\checkmark$ & $\checkmark$ & $\checkmark$ & $\checkmark$ & $\checkmark$ & $\checkmark$ & $\checkmark$ & $\checkmark$ \\
\hline$Z$ veto & - & inverted & - & - & $\checkmark$ & - & - & $\checkmark$ & $\checkmark$ \\
\hline$\Delta R\left(\ell^{ \pm}, \ell^{ \pm}\right)<3.5$ & - & - & - & - & - & - & $\checkmark$ & $\checkmark$ & - \\
\hline$p_{\mathrm{T}}\left(\ell^{ \pm} \ell^{ \pm}\right)>100 \mathrm{GeV}$ & - & - & - & - & - & - & $\checkmark$ & $\checkmark$ & - \\
\hline$\sum\left|p_{\mathrm{T}}(\ell)\right|>300 \mathrm{GeV}$ & - & - & - & - & - & - & $\checkmark$ & $\checkmark$ & - \\
\hline$\Delta M / \bar{M}$ requirement & - & - & - & - & - & - & - & - & $\checkmark$ \\
\hline
\end{tabular}

interaction point (IP) ${ }^{2}$. Leptons which, on the contrary, are produced in the IP are referred to as prompt leptons. Standard Model processes, such as diboson $(V V, V=Z, W)$ or $t \bar{t} V$ production, are also considered and mostly affecting the $4 \ell$ signal region. Backgrounds are studied and constrained in dedicated control and validation regions (see Table 1), which are designed to assess the goodness of the prediction before applying the techniques to the signal region ${ }^{3}$.

\subsection{Background estimation}

As stated, the two major background contributions arise from misreconstructed objects, for which data-driven techniques are preferred over simulation both because of the complexity of the processes involved in particle-detector interaction and of the detector complexity itself. The probability for an electron to be reconstructed with incorrect charge is estimated from a sample of $Z \rightarrow e e$ events, where the ee pair is either opposite- or same-sign [10]. A scale-factor is measured as the ratio between the probabilities measured in data and simulation, and applied to the simulation (mainly Drell-Yan and $t \bar{t}$ events) to correct for its mismodelling. The contribution from events containing at least one fake lepton is estimated relaxing the requirement on lepton isolation or quality, using the fake-factor method [10]. The fake-factor is measured in bins of $p_{\mathrm{T}}$ and can be applied to events with any lepton multiplicity. Lastly, the background composed by events containing prompt leptons (e.g. $V V, t \bar{t} V$ ) is estimated directly from the simulation. The goodness of background prediction is tested in the validation regions (Tab. 1), where a good agreement between data and the total expected background is observed.

\subsection{Systematic uncertainties}

Theory uncertainties which, for most of the simulated samples, account for PDF uncertainties, PDF set and QCD scale variation, are subdominant in most of the analysis regions. The leading

\footnotetext{
${ }^{2}$ A detailed overview on backgrounds from mis-identified objects can be found in Ref. [10].

${ }^{3}$ For example, the DBCR (Tab. 1) is very pure in diboson events and is used to extract the normalization factor for this background from data. The goodness of the extrapolation is tested in the $3 \mathrm{LVR}$ and eventually applied to the $1 \mathrm{P} 3 \mathrm{~L}$ signal region.
} 
experimental uncertainties arise from the data-driven background estimation. The experimental uncertainty related to the charge misidentification probability of electrons arises from the statistical uncertainty of the $Z \rightarrow e e$ sample used in the measurement. They range between $10 \%$ and $20 \%$ as a function of the electron $p_{\mathrm{T}}$ and $\eta$. The experimental uncertainty due to the fake-lepton background is evaluated by varying the nominal fake-factor [9] measurement to account for different fake composition and for the uncertainty on the normalization for simulated events, which need to be subtracted from data when measuring the fake-factor. The uncertainty varies between $10 \%$ to $20 \%$ across all $p_{\mathrm{T}}$ bins.

\subsection{Fit results}

The statistical analysis of the results implements a maximum-likelihood binned fit of the dilepton invariant mass distribution in control and signal regions. The likelihood is defined by the product of a Poisson probability density function describing the observed number of events and Gaussian distributions to constrain nuisance parameters associated with systematic uncertainties. The normalizations for the diboson and for Drell-Yan backgrounds are constrained from control regions and applied to the signal regions. No significant excess is observed in any of the signal regions, as shown in Figure 2.

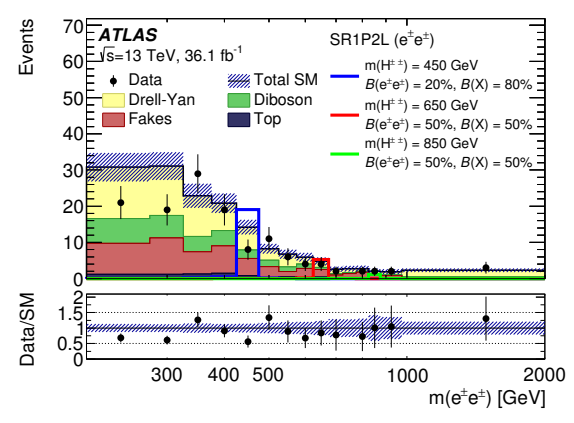

(a)

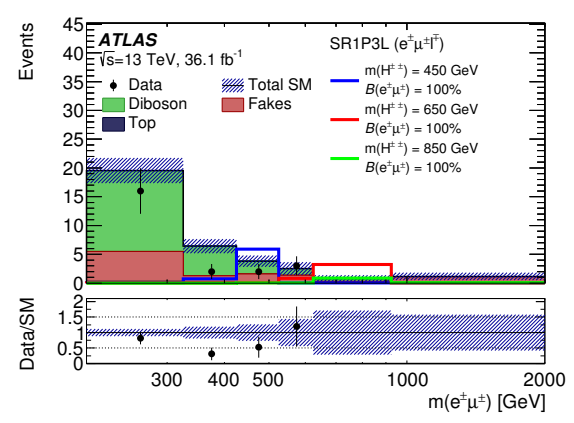

(c)

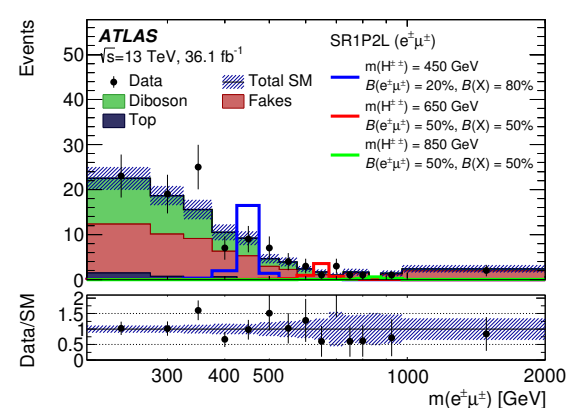

(b)

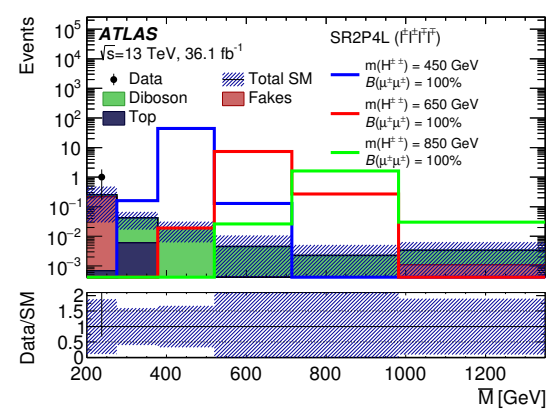

(d)

Figure 2: Comparison between data and simulation in representative signal regions: SR1P2L $\left(e^{ \pm} e^{ \pm}\right)$(a), SR1P2L $\left(e^{ \pm} \mu^{ \pm}\right)$(b), SR1P3L $\left(e^{ \pm} \mu^{ \pm} \ell^{\mp}\right)$ (c) and SR2P4L (d) [9].

In the SR2P4L (Fig. 2d) only one event is observed and found compatible with a $Z Z^{*}$ background event. 


\subsection{Exclusion limits}

The total branching ratio of $H^{ \pm \pm}$can be written as $B\left(H^{ \pm \pm} \rightarrow e^{ \pm} e^{ \pm}\right)+B\left(H^{ \pm \pm} \rightarrow \mu^{ \pm} \mu^{ \pm}\right)+B\left(H^{ \pm \pm} \rightarrow\right.$ $\left.e^{ \pm} \mu^{ \pm}\right)=100 \%$. In this scenario, the sensitivity is dominated by the SR2P4L, which presents the highest $\mathrm{A} \times \varepsilon$. The tested $H^{ \pm \pm}$mass hypotheses vary between 200 and $1300 \mathrm{GeV}$. The results are presented in Figure 3. The fit was performed for values of $B\left(H^{ \pm \pm} \rightarrow \ell^{ \pm} \ell^{ \pm}\right)$from $1 \%$ to $10 \%$ in $1 \%$ intervals, and from $10 \%$ to $100 \%$ in $10 \%$ intervals. The production cross-section is excluded down to $0.1 \mathrm{fb}$, corresponding to 3-4 signal events, which is the theoretical limit of a 95\% CL exclusion.

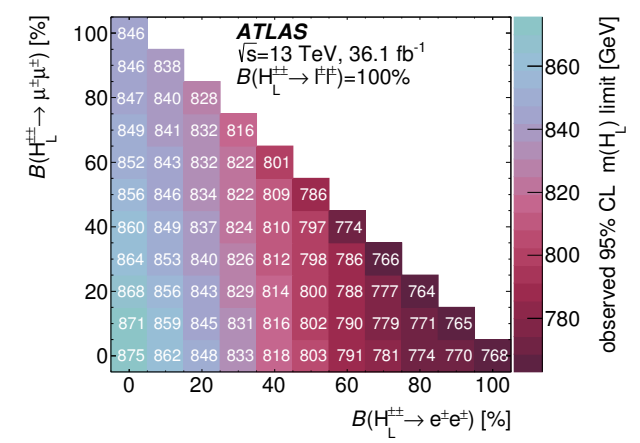

(a)

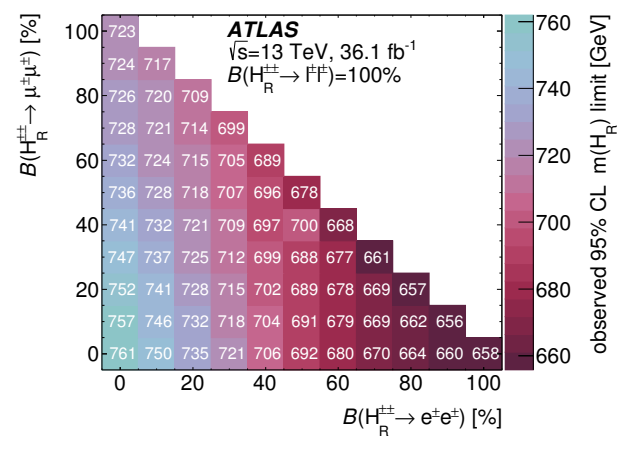

(b)

Figure 3: Observed lower mass limits for the left-handed (a) and right-handed (b) $H^{ \pm \pm}$for all branching ratio combinations that sum to $100 \%$ [9].

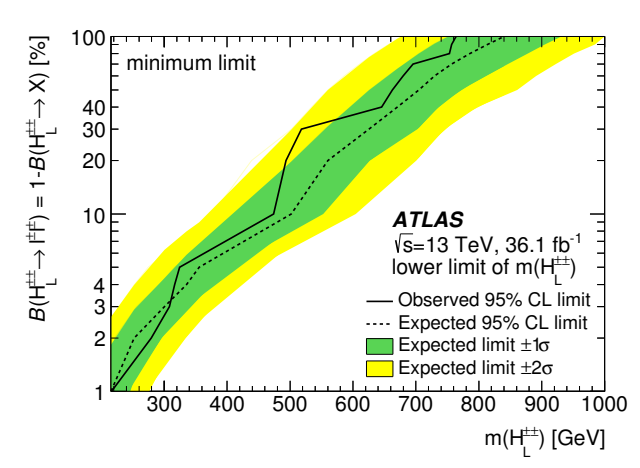

(a)

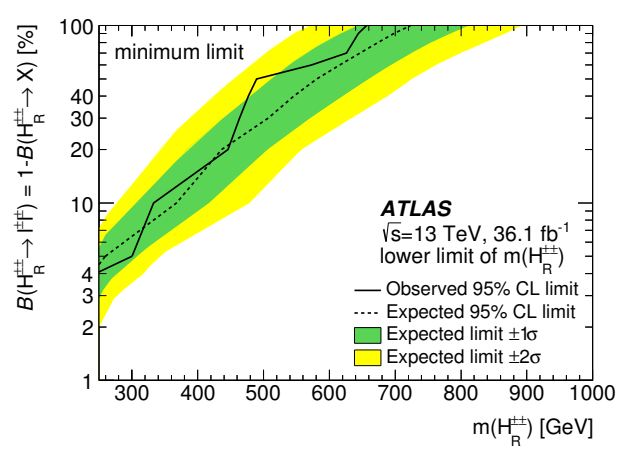

(b)

Figure 4: Lower limit on the $H^{ \pm \pm}$boson mass for the left-handed (a) and the right-handed (b) particle. Plots show the minimum observed limit on the mass as a function of $B\left(H^{ \pm \pm} \rightarrow \ell^{ \pm} \ell^{ \pm}\right)$[9].

Additional motivation to study cases where $B\left(H^{ \pm \pm} \rightarrow \ell^{ \pm} \ell^{ \pm}\right)<100 \%$ is given by type-II SeeSaw models with specific neutrino mass hypotheses, resulting in a fixed branching ratio combination which might differ from $B\left(H^{ \pm \pm} \rightarrow \ell^{ \pm} \ell^{ \pm}\right)=100 \%$. In the latter case, i.e. $B\left(H^{ \pm \pm} \rightarrow\right.$ $\left.\ell^{ \pm} \ell^{ \pm}\right)+B\left(H^{ \pm \pm} \rightarrow X\right)=100 \%, X$ represents $\tau$ leptons or $W$ bosons, as well as particles that escape detection. The branching ratio hypotheses into $B\left(H^{ \pm \pm} \rightarrow e^{ \pm} e^{ \pm}\right), B\left(H^{ \pm \pm} \rightarrow \mu^{ \pm} \mu^{ \pm}\right)$and $B\left(H^{ \pm \pm} \rightarrow e^{ \pm} \mu^{ \pm}\right)$are first considered separately. Figure 4 shows the minimum lower limit among the three flavour decay channels. The observed lower mass limits vary from $770 \mathrm{GeV}$ to $870 \mathrm{GeV}$ for $H_{L}^{ \pm \pm}$with $B\left(H^{ \pm \pm} \rightarrow \ell^{ \pm} \ell^{ \pm}\right)=100 \%$ and are above $450 \mathrm{GeV}$ for $B\left(H^{ \pm \pm} \rightarrow \ell^{ \pm} \ell^{ \pm}\right) \geq 10 \%$. For 
$H_{R}^{ \pm \pm}$the lower mass limits vary from $660 \mathrm{GeV}$ to $760 \mathrm{GeV}$ for $B\left(H^{ \pm \pm} \rightarrow \ell^{ \pm} \ell^{ \pm}\right)=100 \%$ and are above $320 \mathrm{GeV}$ for $B\left(H^{ \pm \pm} \rightarrow \ell^{ \pm} \ell^{ \pm}\right) \geq 10 \%$.

\section{Search for $\boldsymbol{H}^{ \pm \pm} \rightarrow \boldsymbol{W}^{ \pm} \boldsymbol{W}^{ \pm}$}

The search for $H^{ \pm \pm} \rightarrow W^{ \pm} W^{ \pm}$assumes a VEV for the triplet different from zero ${ }^{4}$. The four $W$ boson final state is identified by the presence of light charged leptons, missing transverse energy $\left(E_{\mathrm{T}}\right)$ and jets [11].

Table 2: Preselection criteria for the three channels. In the $3 \ell, \ell_{1} / \ell_{2}$ indicate the same-sign leptons, while $\ell_{0}$ denotes the lepton with a charge opposite to the total lepton charge [11].

\begin{tabular}{|c|c|c|c|}
\hline Selection criteria & $2 \ell^{\mathrm{ss}}$ & $3 \ell$ & $4 \ell$ \\
\hline Trigger & \multicolumn{3}{|c|}{$\begin{array}{l}\text { At least one lepton with } p_{\mathrm{T}}^{\ell}>30 \mathrm{GeV} \\
\text { that fulfils the requirements of single-lepton triggers }\end{array}$} \\
\hline$N_{\ell}\left(L\right.$-type, $\left.p_{\mathrm{T}}>10 \mathrm{GeV},\left|\eta_{\ell}\right|<2.47\right)$ & 2 & 3 & 4 \\
\hline$N_{\ell}\left(T\right.$-type, $\left.p_{\mathrm{T}}>10 \mathrm{GeV},\left|\eta_{\ell}\right|<2.47\right)$ & 2 & $2\left(\ell_{1,2}\right)$ & - \\
\hline$\left|\sum Q_{\ell}\right|$ & 2 & 1 & 0 \\
\hline Lepton $p_{\mathrm{T}}$ threshold & $p_{\mathrm{T}}^{\ell_{1}, \ell_{2}}>30,20 \mathrm{GeV}$ & $p_{\mathrm{T}}^{\ell_{0}, \ell_{1}, \ell_{2}}>10,20,20 \mathrm{GeV}$ & $p_{\mathrm{T}}^{\ell_{1}, \ell_{2}, \ell_{3}, \ell_{4}}>10 \mathrm{GeV}$ \\
\hline$E_{\mathrm{T}}^{\text {miss }}$ & $>70 \mathrm{GeV}$ & $>30 \mathrm{GeV}$ & $>30 \mathrm{GeV}$ \\
\hline$N_{\text {jets }}$ & $\geq 3$ & $\geq 2$ & - \\
\hline$b$-jet veto & \multicolumn{3}{|c|}{$N_{b \text {-jet }}=0$} \\
\hline Low SFOS $m_{\ell \ell}$ veto & - & $m_{\ell^{ \pm} \ell^{\mp}}>15 \mathrm{GeV}$ & $m_{\ell^{ \pm}}{ }_{\ell}>12 \mathrm{GeV}$ \\
\hline$Z$ boson decays veto & $\left|m_{e^{ \pm}} e^{ \pm}-m_{Z}\right|>10 \mathrm{GeV}$ & \multicolumn{2}{|c|}{$\left|m_{\ell^{ \pm} \ell^{\mp}}-m_{Z}\right|>10 \mathrm{GeV}$} \\
\hline
\end{tabular}

Each channel is first selected using the preselection requirements presented in Table 2. These definitions are eventually optimised using a rectangular cut optimization, which depends on the $m_{H^{ \pm \pm}}$hypothesis. The optimization exploits the angular correlation of the $H^{ \pm \pm}$decay products in the $\eta-\phi$-plane, as well as the invariant mass of the system or the $p_{\mathrm{T}}$ of the jets. Some of the variables used in the rectangular cut optimization are presented in Figure 5.

The major background for this search are due to misreconstructed objects and to $V V, t \bar{t} V$ production.

\subsection{Background estimation}

The data-driven techniques used for background estimation are similar to what already discussed Section 2.1. The probability for electron charge misreconstruction, instead of being applied to simulation, is here applied to opposites-sign data events. The fake-factor is also measured similarly to what explained in Section 2.1 however, due to the low number of events in the control region, it is provided as a single number integrated over the lepton $p_{\mathrm{T}}$ spectrum, separately for electrons and muons. The measured fake-factors amount to $F_{e}=0.14 \pm 0.03$ and to $F_{\mu}=0.48 \pm 0.07$ and applied to events containing either two or three leptons [11]. In the $4 \ell$ signal region, where fake

\footnotetext{
${ }^{4}$ The VEV is set $\sim 0.1 \mathrm{GeV}$.
} 


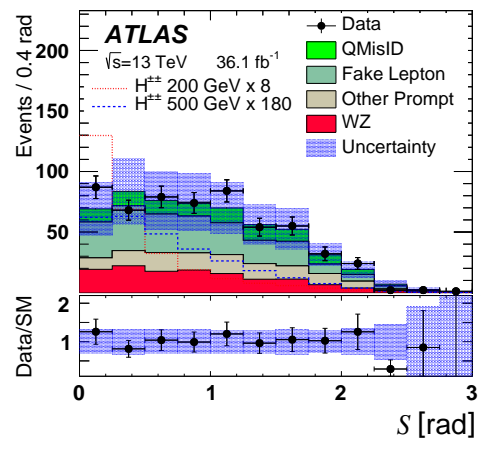

(a)

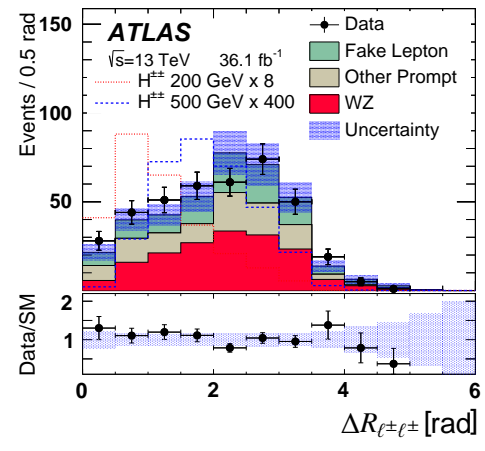

(b)

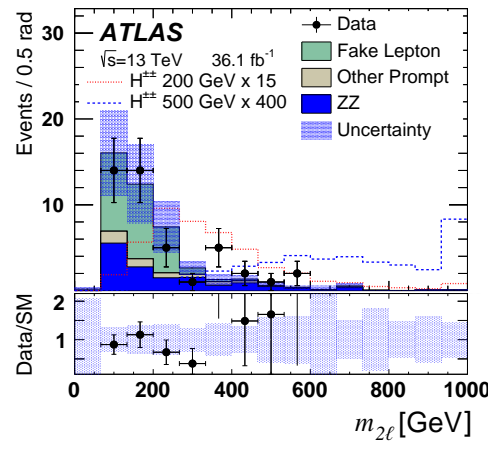

(c)

Figure 5: Distribution of few representative variables used in the optimization of the $2 \ell$ (a), $3 \ell$ (b) and $4 \ell$ (c) signal regions [11].

leptons mostly arise from $b$-jet decays from $t \bar{t} V$ production as well as from light-quarks from $Z+$ jets production, a data-driven scale factor is measured as:

$$
N_{\text {Data } \mid \mathrm{X}}^{\ell}-N_{\text {Prompt } \mid \mathrm{X}}^{\ell}=\lambda_{T}^{\ell} N_{t \overline{\mid} \mid \mathrm{X}}^{\ell}+\lambda_{Z}^{\ell} N_{Z+\mathrm{jets} \mid \mathrm{X}}^{\ell}
$$

where $\mathrm{X}=Z, T$ identifies the light- or heavy-flavour enriched control region. The data-driven scale factors are $\lambda_{T}^{e}=1.12 \pm 0.05, \lambda_{Z}^{e}=1.03 \pm 0.07, \lambda_{T}^{\mu}=1.11 \pm 0.05$ and $\lambda_{Z}^{\mu}=0.94 \pm 0.07$. These factors are then applied as an event weight to the simulation.

\subsection{Systematic uncertainties}

The theoretical uncertainties originate from PDFs, matrix element calculation and parton shower simulation and are found to be $2.4 \%, 1.7 \%$ and $3.8 \%$ respectively for the $2 \ell, 3 \ell$ and $4 \ell$ signal regions [11]. The theoretical uncertainties associated to $V V$ production and $t \bar{t} V$ are assessed using dedicated simulated samples and are $24 \%$ and $17 \%$ respectively. The largest experimental uncertainty arise from electron charge misidentification, which varies between $22 \%$ and $28 \%$ for the $2 \ell$ $e e$ and $e \mu$ channels. The uncertainties of the fake-lepton contribution range from $50 \%$ to $250 \%$ and are mainly due to the low statistic of the control samples.

\subsection{Fit results}

The statistical interpretation of the results implements a likelihood test as discussed in Section 2.3. However, the low number of events in the signal region does not allow the implementation of a multi-bin fit. Therefore, only the total event yield in each of the signal regions is included in the fit, as presented in Figure 6.

\subsection{Exclusion limit}

The expected and observed upper limits on the $H^{ \pm \pm} \rightarrow W^{ \pm} W^{ \pm}$cross-section at $95 \%$ confidence level (CL), obtained from the combination of the $2 \ell, 3 \ell$ and $4 \ell$ channels are presented in Figure 7. The tested $H^{ \pm \pm}$mass hypotheses vary between 200 and $700 \mathrm{GeV}$. Assuming a linear interpolation of the sensitivity between neighbouring mass hypotheses, the observed (expected) lower limit on the mass of the $H^{ \pm \pm}$boson is $220 \mathrm{GeV}(250 \mathrm{GeV})$ at $95 \%$ CL. 


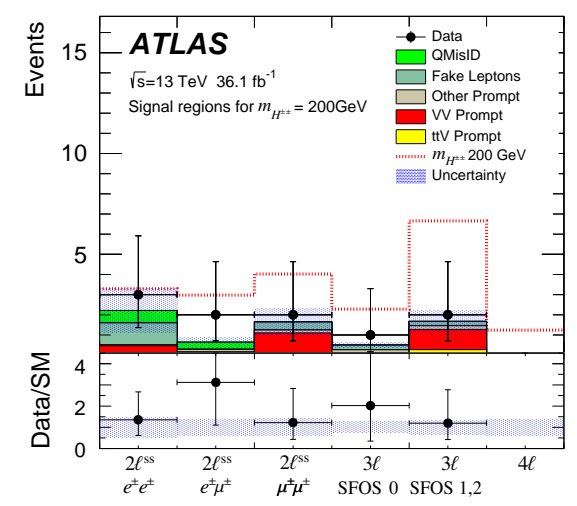

(a)

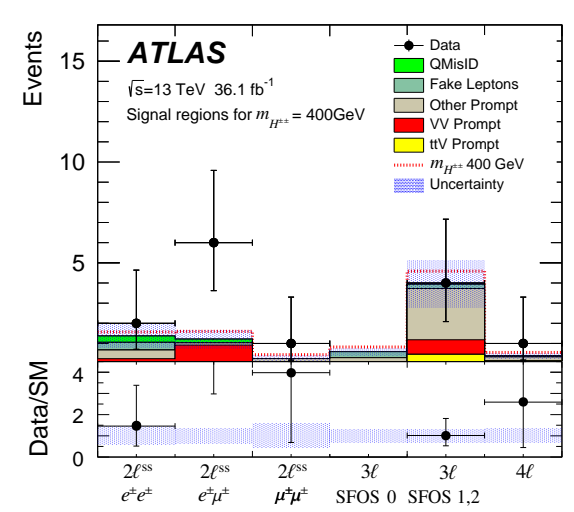

(c)

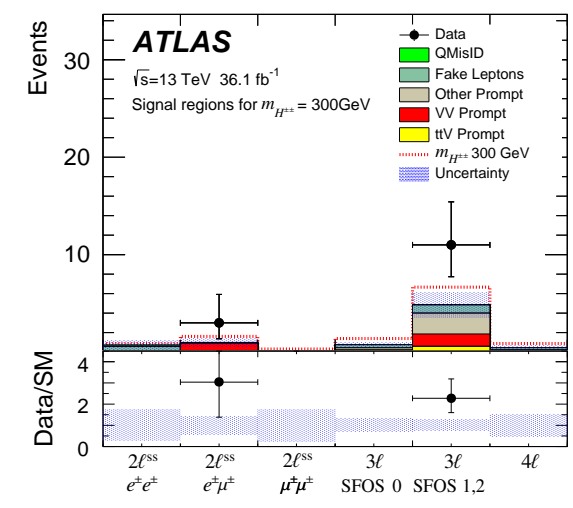

(b)

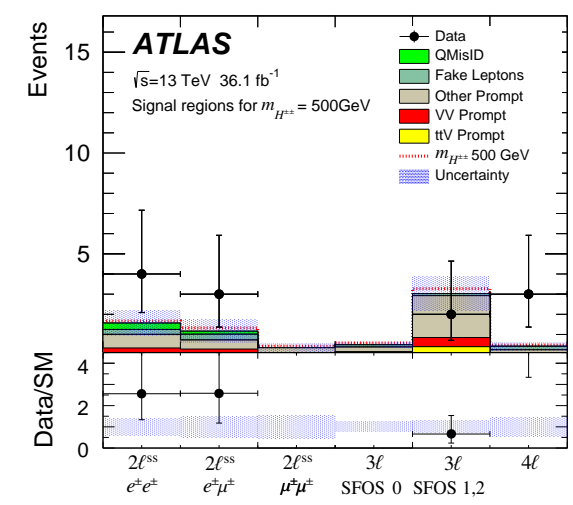

(d)

Figure 6: Event yields in the signal regions, optimised for $m_{H^{ \pm \pm}}=200$ (a), 300 (b), 400 (c), and 500 (d) $\mathrm{GeV}[11]$.

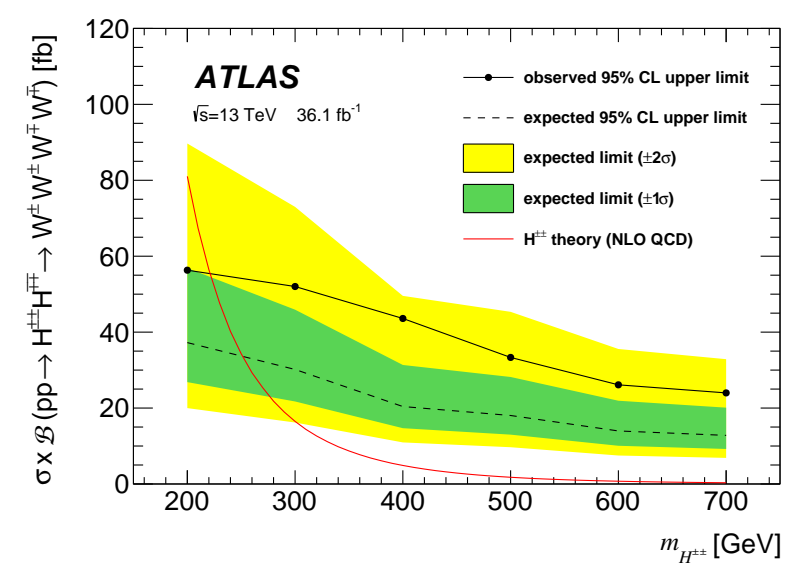

Figure 7: Observed and expected upper limits for $p p \rightarrow H^{ \pm \pm} H^{\mp \mp} \rightarrow W^{ \pm} W^{ \pm} W^{\mp} W^{\mp}$ cross-section times branching ratio at $95 \% \mathrm{CL}[11]$. 


\section{Conclusions}

Two searches for doubly charged Higgs boson production have been presented, using data collected by the ATLAS experiment at $\sqrt{s}=13 \mathrm{TeV}$ and corresponding to an integrated luminosity of 36.1 $\mathrm{fb}^{-1}$. No evidence for doubly charged Higgs boson production was found, however:

- the search for $H^{ \pm \pm} \rightarrow \ell^{ \pm} \ell^{ \pm}$production in left-right symmetric models provides the current best limit on the $H^{ \pm \pm}$mass, due to the high sensitivity of the channels and to the possibility to fully reconstruct the mass of the $H^{ \pm \pm}$boson.

- the search for $H^{ \pm \pm} \rightarrow W^{ \pm} W^{ \pm}$in Higgs triplet models provides lower sensitivity due to the presence of $E_{\mathrm{T}}$ in the final state. This search was performed for the first time in ATLAS.

The foreseen upgrades of both the LHC and the ATLAS experiment, which will allow to collect an integrated luminosity of $300 \mathrm{fb}^{-1}$ by the end of Run 3, will boost the potential for Higgs beyond the Standard Model discoveries.

\section{References}

[1] J. C. Pati and A. Salam, Lepton number as the fourth "color", Phys. Rev. D 10 (1974), 275

[2] P.F. Pérez et al., Neutrino masses and the CERN LHC: Testing the type II seesaw mechanism, Phys. Rev. D78 (2008), 015018

[3] A. Arhrib et al., Higgs potential in the type II seesaw model, Phys. Rev. D 84 (2011), 095005

[4] A. Zee, Quantum numbers of Majorana neutrino masses, Nucl. Phys. B 264 (1986), 99-110

[5] K.S. Babu, Model of “calculable” Majorana neutrino masses, Phys. Lett. B203 (1988), 132-136

[6] H. Georgi, M. Machacek, Doubly charged Higgs bosons, Nucl. Phys. B262 (1985), 463-477

[7] ATLAS Collaboration, The ATLAS Experiment at the CERN Large Hadron Collider, JINST 3 (2008) S08003.

[8] V. Rentala, W. Shepherd and S. Su, A Simplified Model Approach to Same-sign Dilepton Resonances, Phys. Rev. D84 (2011), 035004

[9] ATLAS Collaboration, Search for doubly charged Higgs boson production in multi-lepton final states with the ATLAS detector using proton-proton collisions at $\sqrt{s}=13 \mathrm{TeV}$, Eur. Phys. J. C78 (2018), 199.

[10] G. Ucchielli, Data-driven methods for misreconstructed objects estimation in lepton final states with ATLAS at $\sqrt{s}=13 \mathrm{TeV}$, ATL-PHYS-PROC-2018-088,

https://cds.cern.ch/record/2635886

[11] ATLAS Collaboration, Search for doubly charged scalar bosons decaying into same-sign $W$ boson pairs with the ATLAS detector, CERN-EP-2018-188, Submitted to: Eur. Phys. J.

https://cds.cern.ch/record/2633369 